\section{Biomechanical Prediction of Crack Angle Effect on Local Tensile Stress of Articular Cartilage}

\section{Abstract}

Purpose: Articular cartilage is an important load-bearing structure in synovial joint. Posttraumatic osteoarthritis may occur after cracking insult to cartilage, while not all insult to cartilage could progress into osteoarthritis. The initiating mechanism of osteoarthritis is still not well understood. Tensile stress is considered to have strong relationships with cartilage degradation. We hypothesize that cracks on articular cartilage under compression will induce tensile stress around the crack region, which may lead to chondrocytes change and cartilage degeneration.

Methods: In this study, using a 2D finite element model and animal validation study, the relationship between crack angle and local tensile stress was investigated.

Results: Under pure compressive load, 45 degrees oblique crack on cartilage could induce the highest peak tensile stress (3.9 times to compressive pressure) while no tensile stress was found in perpendicular crack cartilage. Animal study also confirmed finite element prediction that oblique crack may produce type I collagen around crack tip region.

Conclusions: This finding implies that crack angle could be an important factor contributing to initiate osteoarthritis after a traumatic injury. New strategies for prevention and early treatment of osteoarthritis might benefit from this study.

Keywords: Synovial joint; electrolytes; Osteoarthritis; Chondrocytes; Traumatic injury; Type I collagen; Ligaments; Type II collagen

\section{Jia Yu, Meng Zhang, Wen Zhang and Zong-Ping Luo}

Department of Orthopaedics, Medical College, Soochow University, China

Corresponding author: Zong-Ping Luo

\section{” zongping_luo@yahoo.com}

Department of Orthopedics, The First Affiliated Hospital of Soochow University, Orthopedic Institute, Medical College, Soochow University, 708 Renmin Rd, Suzhou, Jiangsu, 215007 China.

Citation: Luo ZP. Biomechanical Prediction of Crack Angle Effect on Local Tensile Stress of Articular Cartilage. J Bone Rep Recommendations. 2016, 2:4.

Received: October 10, 2016; Accepted: October 11, 2016; Published: October 15, 2016

\section{Introduction}

The normal mature articular cartilage is smooth and lubricated, functioning as almost frictionless load-bearing surface in synovial joints, mainly cushioning compressive load. Posttraumatic osteoarthritis (PTOA) may occur after traumatic injury to the joint and around $12 \%$ of the overall symptomatic OA burden can be attributed to PTOA of main joints [1]. However, cartilage is an anural tissue where events such as fibrillation and cracking of its structure cannot be detected by an individual. Besides, a traumatic injury such as intra-articular fracture might reduce its integrity and lead to osteoarthritis [2]. The risk of OA following a significant joint trauma that disrupts the articular surface has been reported to be much higher than the risk of OA in those who did not have a traumatic injury. However, not all the insult to articular cartilage lead to osteoarthritis; less than half of traumatic insult to cartilage would cause osteoarthritis-like changes [3].

An insult such as impact on articular cartilage or intra-articular trauma may produce crack lesions which may lead to a different local stress environment. Alteration of the local mechanical environment around the injury site might determine early $O A$ progress since mechanical factors have been strongly implicated in the development of $O A$, although exact mechanisms of the initial events in cartilage degradation lead to OA are still not elucidated. PTOA degeneration is associated with loss of type II collagen, which is the principle component of the extracellular matrix of articular cartilage, being replaced by type I collagen. Type I collagen is typically found in ligaments and tendons since type I collagen fibrils have enormous tensile strength needed in these structures. Change in tensile stress has been related to osteoarthritis progress but without conclusive evidence. 
Crack lesions are the typical result of insults to articular cartilage [4]. In-depth investigation of early tensile stress of crack lesions could offer insight into OA initiation. We hypothesize that crack on articular cartilage under compression will induce tensile stress around the crack region, which may lead to chondrocytes change and cartilage degeneration. In this study, we will use finite element analysis to explore local tensile stress around crack region and validate simulation predictions with animal model observation.

\section{Methods}

\section{Finite element model}

A simplified two-dimensional rectangular model was generated with Abaqus 6.11 finite element package (Simulia, USA), representing coronal cross-section of articular cartilage of a rabbit distal femur. The $2 \mathrm{D}$ rectangular model size was $4.5 \mathrm{~mm} \times$ $0.50 \mathrm{~mm}$ according to the normal sample size.

A 4-node bilinear plane element (CPE4R) which has quadrilateral strain and reduced integration with hourglass control was used in the model because its good compressive and bending behaviour according to ABAQUS user's manual. The element size was set at $10 \mu \mathrm{m} \times 10 \mu \mathrm{m}$ after model convergence verification. The total mesh number is about 18500 . The rectangular model was assumed as homogenous and isotropic. Elastic Young's modulus $(\mathrm{E}=8 \mathrm{MPa})$ and Poisson ratio $(\mathrm{v}=0.42)$ was assigned according to literature [5]. The bottom edge of rectangle was constrained in vertical direction and the top surface was uniformly compressed with $0.15 \mathrm{MPa}$ pressure to simulate normal loading to knee joint during walking [6].

To simulate a cut-lesion on the articular cartilage, a crack effect was added. The crack length was $300 \pm 25.0 \mu \mathrm{m}$ as it was measured from cross-sectional film of animal knee study. To examine the effect of crack angle, the length was set as $300 \mu \mathrm{m}$ and various crack angles from 0 to 90 degrees, at interval of 15 degrees, were inflicted to the middle node of top surface, as depicted in (Figure 1). The crack itself was set as frictionless contact.

\section{Animal Study}

\section{Animal model}

Four months old New Zealand white rabbits with a weight between 2.5 and $3.0 \mathrm{~kg}$ were obtained from the central animal department breeding facility of the Soochow University. The study was approved by the Institutional Animal Care and Use Committee. They were kept in pairs in cages with sawdust bedding and had free access to a standard diet and tap water.

Fifteen rabbits were randomly divided into 3 groups. Rabbits were anesthetized by chloral hydrate $(0.1 \mathrm{~g} / \mathrm{mL})$ by vein injection with the animals lying supine on the operating table. After shaving the knee joint, the skin of their hind legs was disinfected with entoiodine for $3 \mathrm{~min}$, and then a Para patellar skin incision was made on the medial side of the joint. The patella was then dislocated laterally to expose the fossa intercondyloidea of the distal femur. The crack lesion insult was carefully made in the middle of the femoral trochlear groove using the tip of a shave knife, and the crack lesion was angled in two directions: one perpendicular to the articular surface $\left(90^{\circ}\right)$ and the other oblique $\left(45^{\circ}\right)$. Each rabbit only received one crack lesion. The knee joint was irrigated with $0.9 \%$ sodium solution after surgical operation. The patella was repositioned and the capsule and skin were sutured carefully. After operation procedures, all the animals received antibiotics (penicillin) to prevent infection.

\section{Histology and immunohistochemistry}

For routine histology, animals $(n=5)$ were killed via overdose of chloral hydrate after 2, 6 and 20 weeks. Whole knee joints were dissected and fixed in $10 \%$ neutral buffered formalin for two days. The samples were then dehydrated and embedded in paraffin after decalcification in buffered 10\% EDTA of pH7.4 for 4 weeks. Afterwards, horizontal plane sections of femoral trochlear groove $(5 \mu \mathrm{m})$ were cut into slides by a microtome (RM2165, Leica, Nussloch, Germany).

Type I and type II collagen were then evaluated immunehistochemically. Cartilage slides were deparaffinised in xylene and incubated with testicular hyaluronidase $(2 \mathrm{mg} / \mathrm{mL})$ (H3506-1G, Sigma-Aldrich Co, St. Louis, USA) for $60 \mathrm{~min}$ at 37oC. Afterwards, the slides were treated with $1 \% \mathrm{H} 2 \mathrm{O} 2$ for $30 \mathrm{~min}$ at room temperature (RT) to eliminate endogenous peroxidase activity, and $1.5 \%$ horse serum for $30 \mathrm{~min}$ at RT to eliminate nonspecific protein absorption. The slides were put exposure to the primary antibody at $37^{\circ} \mathrm{C}$. The primary antibodies were anticollagen type I or type II, 1 mg/mL (Ab6308, Abcam, San Francisco, USA), diluted with phosphate-buffered saline. The samples were washed with PBS and used by the secondary antibodies (PK6200, Vector Laboratories, Burlingame, USA) for $60 \mathrm{~min}$ and to avidin at RT. Color was developed with $0.3 \%$ diaminobenzidine tetrahydrochloride (Sk4100, Vector Laboratories, Burlingame, USA).

\section{Results}

Different cracking angles will produce a different tensile stress around the crack tip (Figure 2). The obtained results showed that pure compression load of $0.15 \mathrm{MPa}$ pressure would result into $1.5 \%$ strain at the superior surface of articular cartilage and there was almost uniform strain of $1.5 \%$ of whole intact cartilage. At pure compression, a 90 degrees crack did not induce tensile stress, while the highest peak tensile stress (0.59 MPa, or 3.9 times of compressive pressure loading) was reached with a 45 degrees cracking (Figure 3). With a cracking of 45 degrees, the highest peak tensile strain was predicted, which is $6.81 \%$ or 4.54 times of compressive load strain on the surface of articular cartilage (Figures 4 and 5 ).

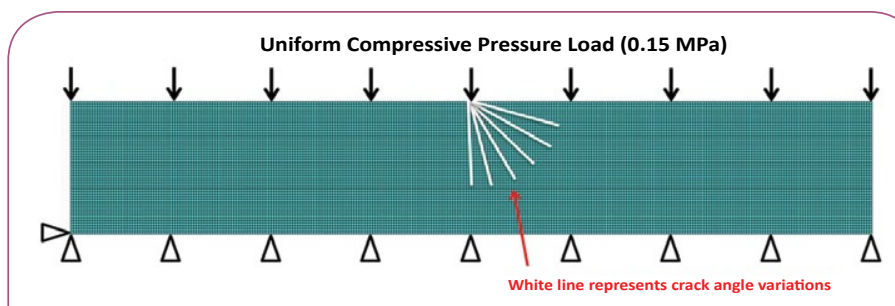

Figure 1 Loading condition for 2D cartilage finite element model. 

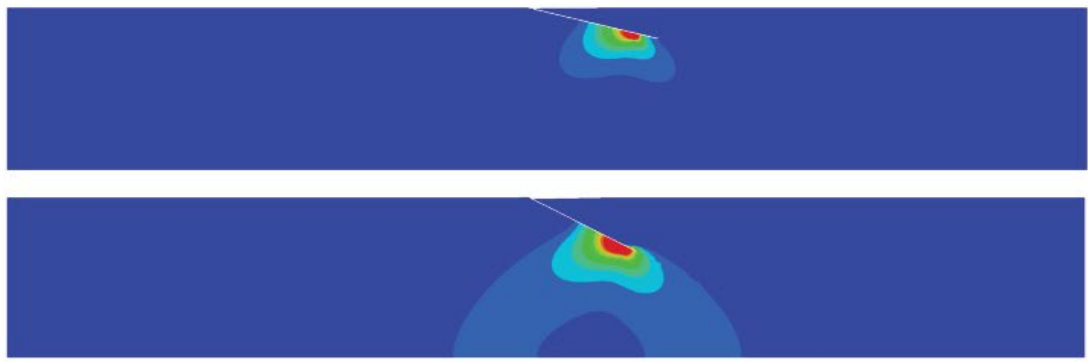

c

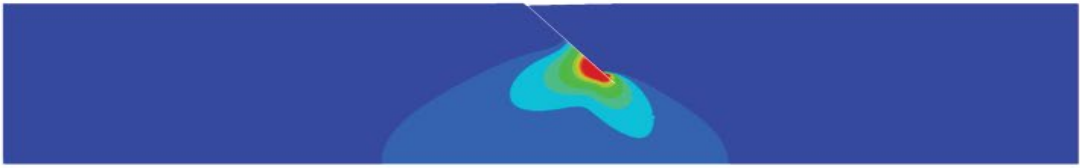

d

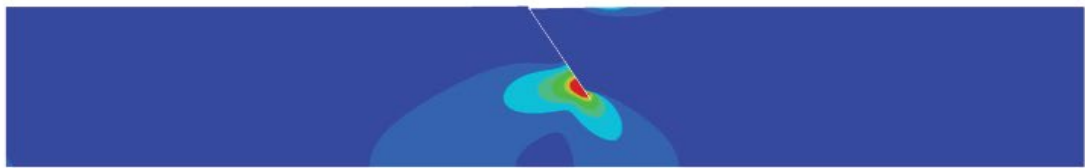

S, Max. In-Plane Principal (Avg: $100 \%$ )

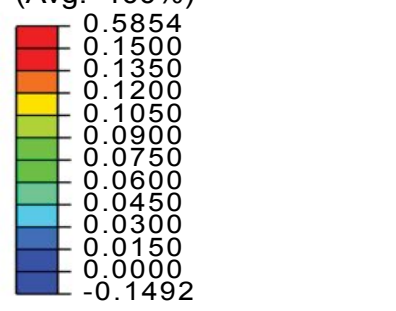

e
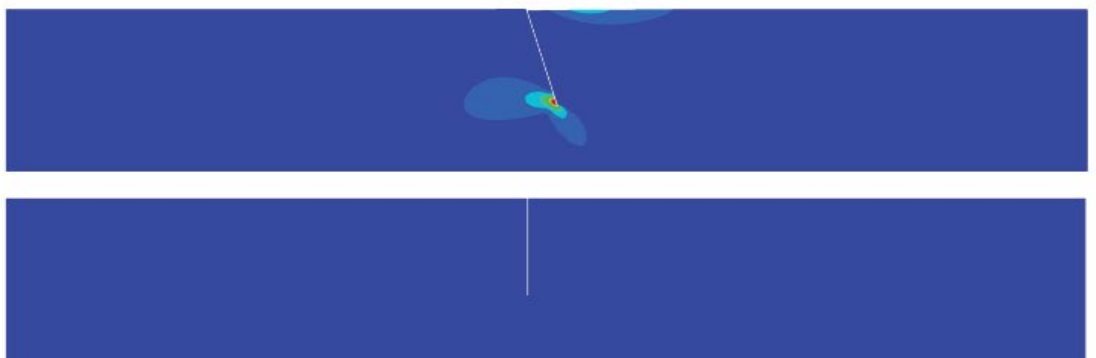

Figure 2 Tensile stress distributions at different crack angles. a. 15 degrees crack. b. 30 degrees crack. c. 45 degrees crack. d. 60 degrees. e. 75 degrees crack. f. 90 degrees crack.

a

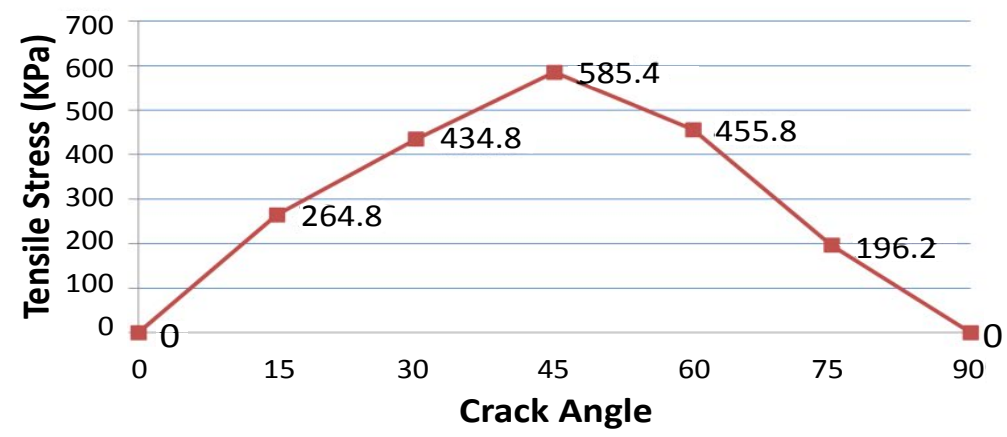

Ratio of Tensile Stress to Compressive Pressure (\%)

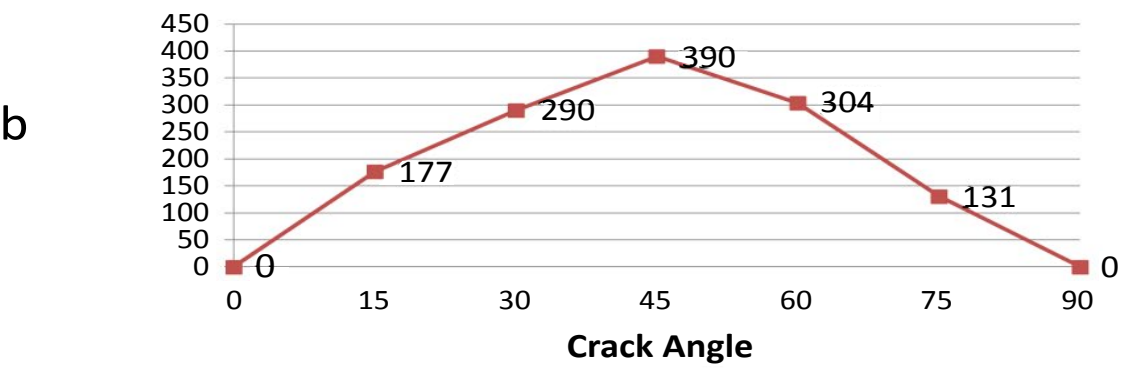

Figure 3 a) Peak tensile stress at different crack angles. b) Ratio of peak tensile stress to compressive pressure varied with different crack angles. 


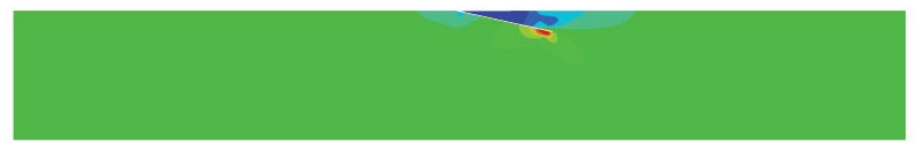

b

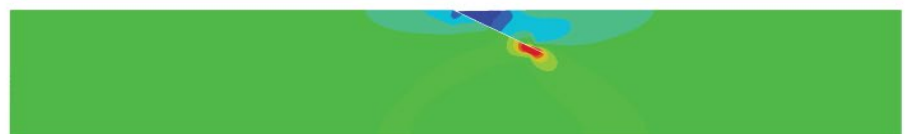

C

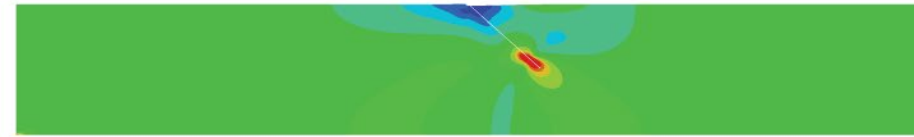

d
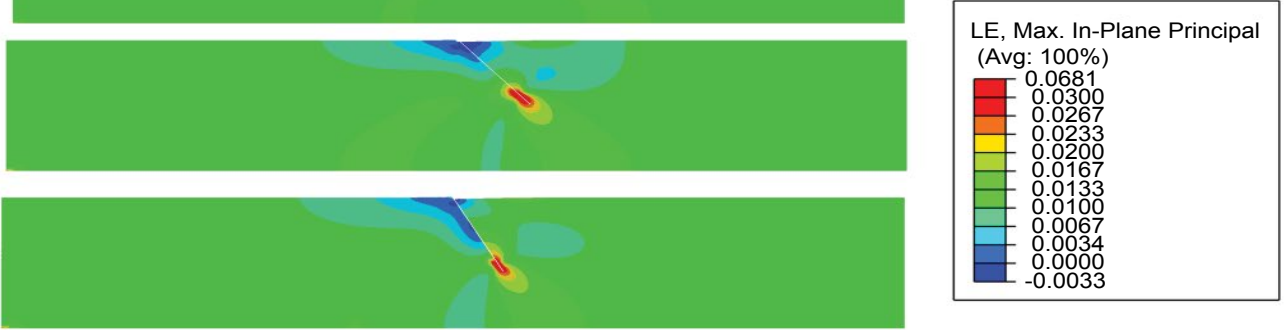

e

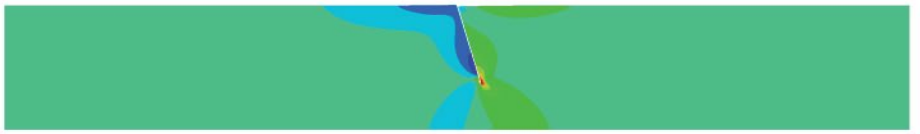

$f$

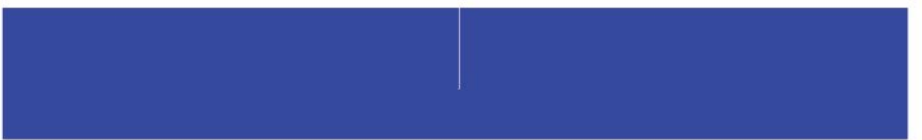

Figure 4

Tensile strain distributions with different crack angles. a) 15 degrees crack. b) 30 degrees crack. c) 45 degrees crack. d) 60 degrees. e) 75 degrees crack. f) 90 degrees crack.

\section{Animal validation}

In the animal study, immunohistochemical results of type I collagen showed the 45 degrees crack had $0.13 \pm 0.07$ of the mean optical density around the crack at 2 weeks increasing progressively without statistically difference to $0.18 \pm 0.05$ at 6 weeks, and to $0.19 \pm 0.09$ at 20 weeks (Figure 6). In comparison, the type II collagen and proteoglycan did not change significantly. At perpendicular crack condition, Type I collagen was not found, and type II collagen and proteoglycan were unchanged at 2, 6 and 20 weeks.

\section{Discussion}

A characteristic feature of $O A$ initiation is cracking, fibrillation, and wear of cartilage; those could be treated as a mechanically driven process. Tensile failure of cartilage has been of particular interest, since it was generally believed that vertical cracks in cartilage were initiated by relatively high tensile stresses on the articular surface. In this study was found that tensile stress has strong relationship with crack angle. The oblique crack insult would induce remarkable tensile stress and increased strain around the crack tip which could influence feedback loops of cartilage degradation process.

Cartilage is composed of fluid, electrolytes, chondrocytes, collagen fibers, proteoglycans and other glycoproteins $[5,7,8]$. The dense collagen fiber network provides load-bearing support and tensile strength to the diarthrodial joints. OA is the progress of degradation and loss of articular cartilage, subchondral bone remodeling, and chronic inflammation of the synovial membrane.
Its etiology, although is not well understood. OA is considered to result from a complex system of interacting mechanical, biological, biochemical, molecular and enzymatic feedback loops [9].

While studies via computational models of joint contact show that the tensile stress on the surface is quite low, still some tensile stress exists within the cartilage. Under normal physiologic condition, articular cartilage would have 1-3\% compressive strain [10] and normal synovial joints have a relatively low coefficient of friction of about 0.001 . In this study, with a compressive strain set at $1.5 \%$ (which is within the normal range), we found that under pure compression load without any shear stress, the oblique crack could induce tensile stress around crack tip as well. This finding implies that crack angle could be a contributing factor to initiate OA after a traumatic injury.

Previous studies have showed that 2D FE models can simultaneously predict stress distribution and reaction forces during swelling, confined compression, indentation and unconfined compression of articular cartilage. In this study, 2D model was considered to be accurate enough to predict stress under pure compression. Other studies related to torsion and shear loading could benefit of implementation of 3D models.

This study present some disadvantages. The mechanical response of cartilage has relationship with the flow of fluid through the tissue. When deformed, fluid flows through the cartilage and across the articular surface. Recognizing that fluid flow and deformation are interdependent and has led to the modeling of cartilage as a mixture of fluid and solid components, which is 
a biphasic model $[11,12]$. Under pure compression static load, single-phase elastic solid model is relative accurate to describe the behavior of cartilage with crack insult. Thus, considering the applied loading condition, we did not consider the inclusion of fluid into this quasi-static model.

The cartilage matrix is occupied by proteoglycan aggregates, which is limited by the entangling collagen framework. When cartilage is compressed by loads, the negatively charged sites on aggrecan are pushed closer together, which increases their mutual repulsive force and adds to the compressive stiffness of the cartilage [13]. Damage to the collagen framework also reduces the compressive stiffness of the tissue, since the aggregated proteoglycans are contained less efficiently. Type $X$ collagen has been shown to be up-regulated in osteoarthritic cartilage, and

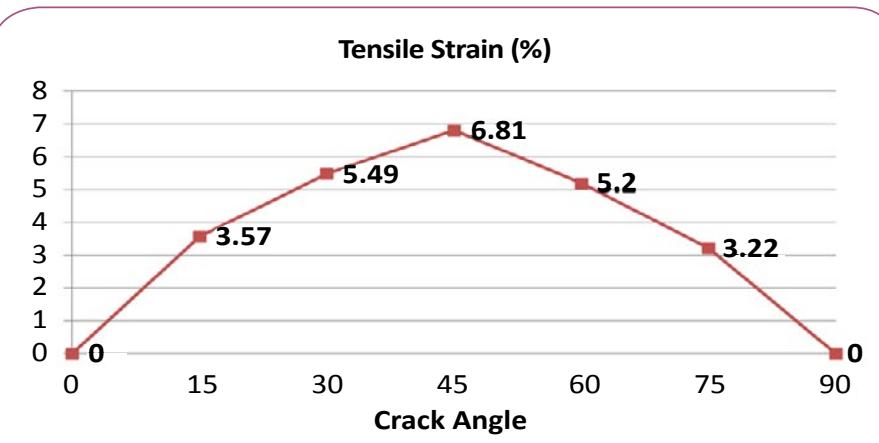

Figure 5 Peak tensile stress varied with different crack angles. also by mechanical loading in normal cartilage [14]. In this study, detailed micro structural of cartilage was not modeled, therefore the effect of aggregated proteoglycans and constraint of fiber on tensile stress were not considered, which may underestimate maximum tensile stress in cartilage.

In this study animal models of cut insult on articular cartilage were used. Animal models are used to provide a controlled environment for studying the progression of OA. Although OA may be induced by numerous means, models based on disruption of the mechanical environment of the joint, either by surgical alteration of periarticular structures or by abnormal joint load, are commonly used and benefit from its implementation [15].

\section{Conclusion}

Oblique cracks may remarkably induce local tensile stress to amplitudes of several times of loading and could lead to formation or type I collagen. This finding suggests that tensile stress is sensitive to crack angle on articular cartilage, which might be one of the mechanism to explain the initiation of PTOA.

\section{Conflicts of Interest Statement}

The authors hereby certify that the work which is reported in the manuscript has not received financial support from any company or other commercial source, and the authors do not have any special financial interest with other organization.
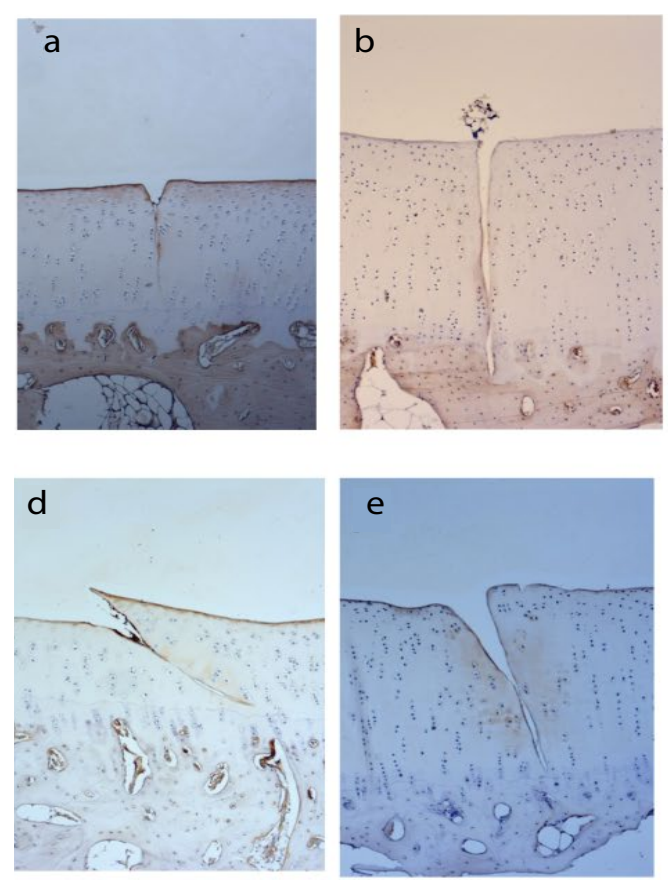
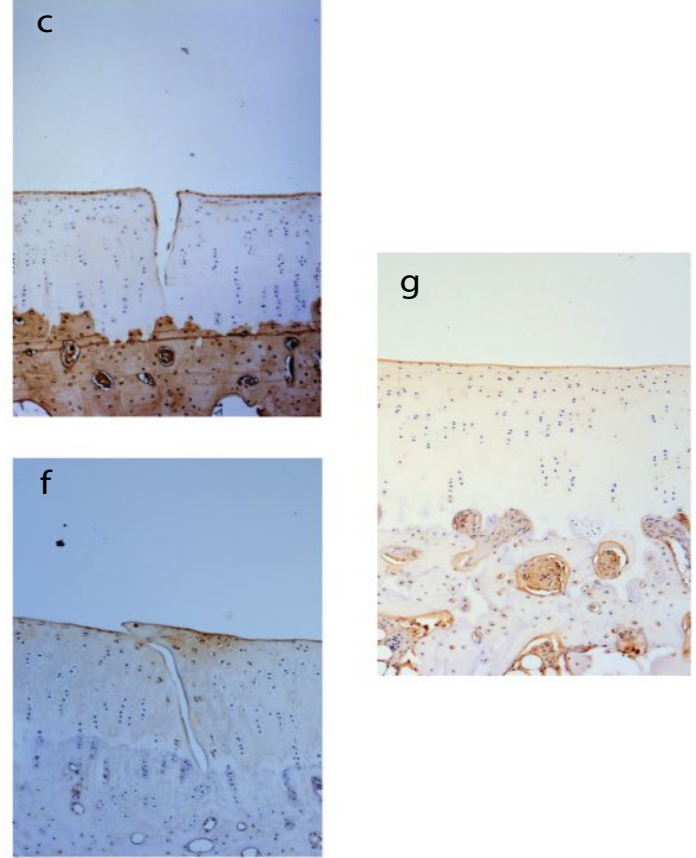

Type I Collagen

Figure 6 Immunohistochemical staining of type I collagen showed increase from 2 weeks to 20 weeks around the 45 degrees crack but no presentation around the 90 o crack. a) 45 degrees crack at 2 weeks. b) 45 degrees crack at 6 weeks. c) 45 degrees crack at 20 weeks. d) 90 degrees crack at 2 weeks. e) 90 degrees crack at 6 weeks. f) 90 degrees crack at 20 weeks. 


\section{Acknowledgments}

This work was supported by the National Natural Science
Foundation of China (81320108018, 31270995 and 11572211), the Priority Academic Program Development of Jiangsu Higher Education Institutions (PAPD). 


\section{References:}

1 Brown TD, Johnston RC, Saltzman CL, Marsh JL, Buckwalter JA (2006) Posttraumatic osteoarthritis: a first estimate of incidence, prevalence, and burden of disease. J Orthop Trauma 20: 739-744.

2 Hunter W. (1995) Of the structure and disease of articulating cartilages. Clin Orthop Relat Res 317: 3-6.

3 Anderson DD, Chubinskaya S, Guilak F, Martin JA, Oegema TR, et al. (2011) Post-traumatic osteoarthritis: Improved understanding and opportunities for early intervention. J Orthop Res 29: 802-809.

4 Thambyah A, Zhang G, Kim W, Broom ND (2012) Impact induced failure of cartilage-on-bone following creep loading: a microstructural and fracture mechanics study. J Mech Behav Biomed Mater 14: 239247.

5 Julkunen P, Harjula T, livarinen J, Marjanen J, Seppanen K, et al. (2009) Biomechanical, biochemical and structural correlations in immature and mature rabbit articular cartilage. Osteoarthritis Cartilage 17: 1628-1638.

6 Coughlin KM, Peura GD, Fleming BC, Hallock S, Beynnon BD (2005) In vivo loads in the medial compartment of the rabbit knee. Clin Biomech 20: 1007-1009.

7 Huang C-Y, Stankiewicz A, Ateshian GA, Mow VC (2005) Anisotropy, inhomogeneity, and tension compression nonlinearity of human glenohumeral cartilage in finite deformation. J Biomech 38: 799-809.

8 Mow V, Kuei S, Lai W, Armstrong C (1980) Biphasic creep and stress relaxation of articular cartilage in compression: theory and experiments. J Biomech Eng 102: 73-84.

9 Martel-Pelletier J, Boileau C, Pelletier J-P, Roughley PJ (2008) Cartilage in normal and osteoarthritis conditions. Best Pract Res Clin Rheumatol 22: 351-384.

10 Buckwalter JA, Mankin HJ, Grodzinsky AJ (2005) Articular cartilage and osteoarthritis. Instructional Course Lectures-American Academy of Orthopaedic Surgeons 54: 465.

11 Mow VC, Holmes MH, Lai WM (1984) Fluid transport and mechanical properties of articular cartilage: a review. J Biomech 17: 377-394.

12 Julkunen P, Harjula T, Marjanen J, Helminen HJ, Jurvelin JS (2009) Comparison of single-phase isotropic elastic and fibril-reinforced poroelastic models for indentation of rabbit articular cartilage. J Biomech 42: 652-656.

13 Kaukinen $A$, Laasanen $M$, Lammentausta $E$, Halmesmäki $E$, Helminen $\mathrm{H}$, et al. (2005) Destructive testing of articular cartilage in compression-effect of collagen network.

$14 \mathrm{Wu} Q Q$, Chen Q (2000) Mechanoregulation of chondrocyte proliferation, maturation, and hypertrophy: ion-channel dependent transduction of matrix deformation signals. Exp Cell Res 256: 383391.

15 Stoop R, Buma P, Van Der Kraan P, Hollander A, Billinghurst RC, et al. (2001) Type II collagen degradation in articular cartilage fibrillation after anterior cruciate ligament transection in rats. Osteoarthritis Cartilage 9: 308-315. 